\title{
Elimination of glucose contamination from adipocyte glycogen extracts
}

\author{
Patricia M. Nunes, ${ }^{a}$ Eugénia Carvalho ${ }^{\mathrm{a}}$ and John G. Jones ${ }^{\mathrm{a}, \mathrm{b}, *}$ \\ ${ }^{a}$ Center for Neurosciences and Cell Biology, Department of Zoology, University of Coimbra, 3004-517 Coimbra, Portugal \\ ${ }^{\mathrm{b}}$ NMR Research Unit, Department of Biochemistry, Faculty of Sciences and Technology, Apartado 3126, \\ University of Coimbra, 3001-401 Coimbra, Portugal
}

Received 5 December 2007; received in revised form 19 February 2008; accepted 1 April 2008

Available online 7 April 2008

\begin{abstract}
Glycogen was quantified in rat adipocytes by isolation using conventional KOH digestion and ethanol precipitation, followed by hydrolysis and spectrophotometric assay of the glucose product. A concentration of $0.193 \pm 0.020 \mu \mathrm{mol}$ glucosyl units/ $10^{6}$ cells was recorded. When this procedure was modified by including a $4 \mathrm{~h}$ incubation with glucose oxidase prior to glycogen hydrolysis, the glycogen concentration was found to be $0.055 \pm 0.008 \mu \mathrm{mol}$ glucosyl units $/ 10^{6}$ cells. Therefore in adipocytes, conventional glycogen assays give substantial overestimates due to incomplete removal of glucose during glycogen isolation. Contaminant glucose can be scavenged in a simple manner by incubation with glucose oxidase prior to glycogen hydrolysis.

(c) 2008 Elsevier Ltd. All rights reserved.
\end{abstract}

Keywords: Adipocyte; Glycogen extraction; Glucose oxidase; Amyloglucosidase

In addition to liver and muscle, a wide range of other tissues can synthesize and mobilize glycogen including brain, chondrocytes, and adipocytes. ${ }^{1-3}$ Compared to liver and muscle, the concentrations of glycogen in these tissues are typically much smaller. There is evidence that these small glycogen pools may play a key role as a substrate buffer to counter the effects of fluctuating glucose supply and demand in neurons ${ }^{3}$ and possibly other cell types. As a result, there is renewed interest in the precise quantification of glycogen in these tissues. The Achilles heel of the conventional glycogen assay method is the separation of glycogen from cell or medium glucose. Since glycogen is typically quantified in terms of glucose units following amyloglucosidase hydrolysis, incomplete removal of glucose during purification will result in an overestimate of glycogen. The potential for this error is increased when the glycogen concentrations are low relative to glucose. The contaminant glucose can be

\footnotetext{
Abbreviations: KR, Krebs Ringer

* Corresponding author. Tel.: +351 239824 531; fax: +351 239853

607; e-mail: john.jones@netc.pt
}

selectively removed from the isolated glycogen preparation by the addition of glucose oxidase. This enzyme requires no cofactors or substrates to convert glucose to gluconate, only oxygen. Glucose oxidase also functions well in the same buffer medium used for the subsequent amyloglucosidase hydrolysis of glycogen to glucose hence there is no need for buffer adjustment or replacement.

Given the amount of glycogen present in adipocyte preparations $(\sim 0.1 \mu \mathrm{mol})$, a minor amount of glucose $(>10 \mathrm{nmol})$ would result in a significant contamination. Therefore, all reagents and extraction steps were examined for the presence or generation of glucose. The amyloglucosidase enzyme preparation did not contain detectable amounts of glucose as assayed by ${ }^{1} \mathrm{H}$ NMR and enzymatic assay. When the $\mathrm{KOH}$ extraction procedure was performed on solutions containing $7.5 \mathrm{mg}$ of commercial glycogen $(42 \mu \mathrm{mol}$ of glucose equivalents or 400 times the amount of glycogen in a typical preparation), glucose was not detected by enzymatic assay and ${ }^{1} \mathrm{H}$ NMR analysis showed only signals from glycogen. Therefore, the glycogen extraction procedure per se does not result in the appearance of glucose. The glucose 
oxidase enzyme preparation was also analyzed by ${ }^{1} \mathrm{H}$ NMR and no glucose or gluconate signals were detected, hence its addition to the extraction procedure does not introduce glucose. To demonstrate the efficacy of glucose removal, the extraction procedure and glucose oxidase treatment were performed on a solution containing $7 \mathrm{mg}$ of commercial glycogen $(39 \mu \mathrm{mol}$ glucose equivalents) and $20 \mathrm{mg}(111 \mu \mathrm{mol})$ of free glucose. ${ }^{1} \mathrm{H}$ NMR spectra were taken before and after the addition of glucose oxidase (Fig. 1). The glucose ${ }^{1} \mathrm{H}$ signal was reduced by $>99 \%$ following glucose oxidase treatment.

Eleven batches of primary culture adipocytes were prepared as described in the methods. Glycogen was extracted by the $\mathrm{KOH} /$ ethanol method and the glycogen pellet was re-suspended in acetate buffer. A known portion of this solution was hydrolyzed to glucose by amyloglucosidase, and the glucose was assayed. Under these conditions, the glycogen concentration, expressed as glucosyl equivalents, was estimated to be $0.193 \pm 0.020 \mu \mathrm{mol}$ glucosyl units $/ 10^{6}$ cells, equivalent to $232 \mu \mathrm{g}$ of glycogen/gram of adipose tissue. To determine the efficacy of glucose oxidase for removing glucose under these conditions, the hydrolyzed preparation was treated with glucose oxidase and aerated as

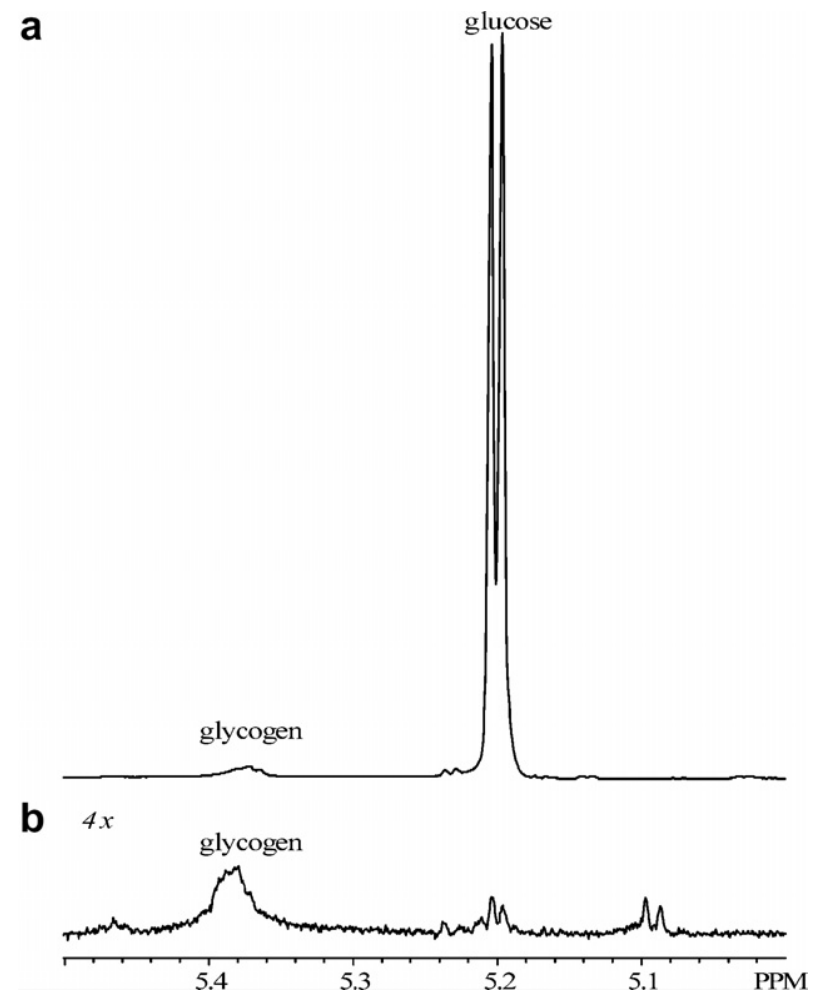

Figure 1. ${ }^{1} \mathrm{H}$ NMR spectra of a solution of $7 \mathrm{mg}$ glycogen and $20 \mathrm{mg}$ glucose in $5 \mathrm{~mL}$ of sodium acetate buffer before (a) and after (b) the addition of glucose oxidase. The region from 5.0 to $5.5 \mathrm{ppm}$ featuring the glucose hydrogen $1 \alpha$ and glycogen hydrogen 1 signals is shown. Due to the limited solubility of glycogen in this sample, its ${ }^{1} \mathrm{H}$ NMR signal does not represent the total amount of glycogen present. described in the methods. The glucose concentration after glucose oxidase treatment was found to be $0.012 \pm 0.005 \mu \mathrm{mol} / 10^{6}$ cells, indicating that $\sim 95 \%$ of glucose had been removed by glucose oxidase under these conditions. The remaining portion of the intact glycogen preparation was incubated with glucose oxidase to scavenge any contaminant glucose, and glucose oxidase was then denatured by boiling the solution. Glycogen was subsequently hydrolyzed with amyloglucosidase, and the released glucose was assayed to obtain glycogen levels. Under these conditions, the glycogen concentration was estimated to be $0.055 \pm 0.008 \mu \mathrm{mol}$ glucosyl units $/ 10^{6}$ cells, or $66 \mu \mathrm{g} / \mathrm{gram}$ of adipose tissue (Fig. 2), less than one-third of the initial value.

Reference values for rat adipocyte glycogen levels show a considerable range of values depending on the dietary regime, or whether the adipocytes were harvested from fed or fasted animals. In adipocytes derived from $24 \mathrm{~h}$ fasted rats, glycogen levels of $48 \mu \mathrm{g} / \mathrm{gram}$ of adipose tissue were reported. ${ }^{5}$ In animals that were sacrificed in the absorptive state (i.e., immediately after overnight feeding), adipocyte glycogen levels of $323 \mu \mathrm{g} / \mathrm{g}$ wet weight were reported. ${ }^{6}$ Animals that were adapted to meal-feeding for 7 days and were sacrificed immediately after the meal had adipocyte glycogen levels of $872 \mu \mathrm{g} / \mathrm{g}$ wet weight. ${ }^{6}$ Since the animals in our study were sacrificed in the early postabsorptive state (i.e., $\sim 2 \mathrm{~h}$ after overnight feeding), the most pertinent reference value for comparison is the $323 \mu \mathrm{g} / \mathrm{g}$ wet weight reported for absorptive animals. This value is similar to our uncorrected estimate of $232 \mu \mathrm{g} / \mathrm{g}$ wet weight, while our corrected estimate of $66 \mu \mathrm{g} / \mathrm{g}$ wet weight is comparable to the $48 \mu \mathrm{g} / \mathrm{gram}$ reported for $24 \mathrm{~h}$ fasted animals.

Our results demonstrate that the standard method of glycogen extraction and isolation does not completely

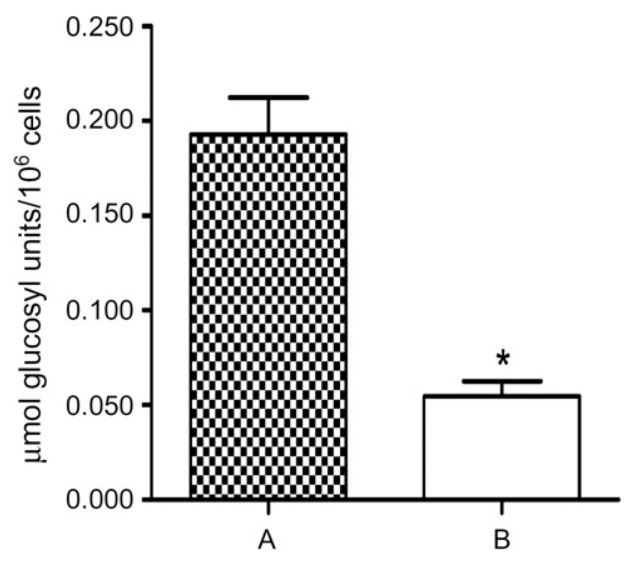

Figure 2. Quantification of glycogen following the isolation and the hydrolysis of the isolated glycogen to glucose by a standard extraction procedure (A) and by the addition of the glucose oxidase incubation step prior to glycogen hydrolysis (B). Data and error bars represent the mean and standard error of 11 adipocyte preparations. $*=p<0.001$ compared to the standard procedure. 
exclude glucose. With tissues that contain high glycogen levels such as liver and muscle, a residual amount of glucose may not significantly alter the estimates of glycogen concentrations. However, with adipocytes (and possibly other cells or tissues with low glycogen levels), the presence of even a residual amount of glucose in the isolated glycogen preparation can significantly inflate the estimates of glycogen concentration. Glucose oxidase treatment provides a convenient and quantitative means for scavenging residual glucose before glycogen is hydrolyzed.

\section{Experimental}

\subsection{General methods}

Sodium chloride, sodium sulfate, potassium chloride, potassium hydroxide, di-hydrogen potassium phosphate, magnesium sulfate, D-Glucose, HEPES, BSA fatty acid free (Fraction V), perchloric acid, glucose oxidase type X-s (Aspergillus niger, Sigma-Aldrich, Portugal) and amyloglucosidase (A. niger, containing $<0.02 \%$ glucose, Sigma-Aldrich, Portugal), Collagenase A (Clostridium histolyticum, Roche Diagnostics, USA), and ethanol $99 \%$ (v/v) were analytical grade and used without further purification.

\subsection{Adipocyte isolation and culture}

All the experiments were conducted according to EU guidelines on ethical use of experimental animals (86/ 609/EEC). Five male Wistar Han eight weeks old (250-300 g) were purchased from Charles River Laboratories S.A., Spain. Animals were rapidly killed by cervical dislocation, and fat pad tissue was rapidly removed to a $37{ }^{\circ} \mathrm{C}$ modified Krebs Ringer (KR) buffer in $\mathrm{mM}$ $\left(120 \mathrm{NaCl}, 4.8 \mathrm{KCl}, 1.2 \mathrm{KH}_{2} \mathrm{PO}_{4}, 0.6 \mathrm{MgSO}_{4}, 1.2 \mathrm{CaCl}_{2}\right.$ 2 HEPES, 5.5 Glucose) containing $200 \mathrm{nM}$ adenosine and BSA $40 \mathrm{mg} / \mathrm{mL}$. Isolated fat cells were prepared by collagenase $(0.8 \mathrm{mg} / \mathrm{mL})$ digestion of minced fat tissue in a $37^{\circ} \mathrm{C}$ shaking water bath. After $40 \mathrm{~min}$, cells were filtered through a nylon mesh with size pore $400 \mu \mathrm{m}$, washed three times with KR buffer without glucose. Cells were then re-suspended in ten volumes of KR. Cell size and numbers were measured as in the previous description. ${ }^{4}$ Each extraction was performed with an average of $0.6 \times 10^{6}$ cells.

\subsection{Glycogen extraction}

Cell suspension was digested for 30 min with a $3 \mathrm{~mL}$ of $\mathrm{KOH}$ solution $30 \%(\mathrm{w} / \mathrm{v})$ at $70{ }^{\circ} \mathrm{C}$. One volume of sodium sulfate $6 \%(\mathrm{w} / \mathrm{v})$ was added to co-precipitate the glycogen and $10 \mathrm{~mL}$ of pure ethanol was also added to ensure total glycogen precipitation. Glycogen samples were stored overnight at $4{ }^{\circ} \mathrm{C}$ and then centrifuged for $5 \mathrm{~min}, 5000 \mathrm{rpm}$ at same temperature. Supernatants were removed and glycogen pellets were re-suspended in $5 \mathrm{~mL}$ water and neutralized, and aliquots were taken. Glycogen suspensions were dried to eliminate ethanol residues, and re-suspended in $5 \mathrm{~mL}$ acetate buffer $10 \mathrm{mM}$ (pH 5). Glucose was oxidized to gluconate by adding 100-200 units of glucose oxidase. The samples were air bubbled for $4 \mathrm{~h}$, and then the enzyme was denatured by heating for $5 \mathrm{~min}$ at $100^{\circ} \mathrm{C}$. For glucose assays, aliquots were taken and any protein present was removed with perchloric acid precipitation. The $\mathrm{pH}$ was readjusted to 7 and insoluble salts were removed by centrifugation. Glycogen hydrolysis was performed by incubation with 20 units of amyloglucosidase for $2 \mathrm{~h}$ at $55^{\circ} \mathrm{C}$. Aliquots were also kept. These aliquots were submitted to protein denaturation by adding perchloric acid and removing pellets after a short centrifugation. Supernatants were neutralized, and salts were removed by a new centrifugation.

\subsection{Glucose assays}

A simple colorimetric glucose assay kit (Invitrogen, Spain) based on glucose oxidase reaction was used to measure glucose in all aliquots: initial contamination, after enzymatic activities, glucose oxidase and amyloglucosidase. A microplate absorbance reader (Spectramax Plus 384, Molecular Devices, USA) was used in $560 \mathrm{~nm}$ wave length. By making use of one calibration curve, glucose concentrations were calculated for all the steps. Commercial glycogen samples $(1.5 \mathrm{mg} / \mathrm{mL})$ were submitted to the same treatment to determine whether either the $\mathrm{KOH}$ extraction procedure or the glucose oxidase incubations caused the release of glucose from glycogen. This procedure was performed four times. ${ }^{1} \mathrm{H}$ NMR spectra of glucose and glycogen mixtures taken before and after the addition of glucose oxidase were acquired with a Varian Unity $500 \mathrm{MHz}$ system and $5-\mathrm{mm}$ probe. The water signal was suppressed with a standard pre-saturation pulse sequence. Chemical shifts are reported as ppm downfield of TMS.

\subsection{Statistical analysis}

All the results are presented as mean \pm standard error (S.E.). Student's unpaired two-tailed $t$-test was used to compare the data, and differences were considered significant when $p$ values were less than 0.05 .

\section{Acknowledgment}

This work was supported by a grant from the Portuguese Foundation of Science and Technology (POCI SAU-MMO/57598/2004). 


\section{References}

1. Hutchison, M. R.; Bassett, M. H.; White, P. C. Endocrinology 2007, 148, 3122-3130.

2. Jurczak, M. J.; Danos, A. M.; Rehrmann, V. R.; Allison, M. B.; Greenberg, C. C.; Brady, M. J. Am. J. Physiol. Endocrinol. Metab. 2007, 292, E952-E963.
3. Gruetter, R. J. Neurosci. Res. 2003, 74, 179-183.

4. Smith, U.; Sjostrom, L.; Bjorntorp, P. J. Lipid Res. 1972, 13, 822-824.

5. Armstrong, M. K.; Romsos, D. R.; Leveille, G. A. J. Nutr. 1976, 106, 884-891.

6. Leveille, G. A. J. Nutr. 1966, 90, 449-460. 